\title{
A comparative study for the assessment of Ikonos satellite image-fusion techniques
}

\author{
Javier Medina ${ }^{1,2}$, Nelson Vera $^{2}$, Erika Upegui ${ }^{1,2}$ \\ ${ }^{1}$ Department of Cadastral Engineering and Geodesy, School of Engineering, Universidad Distrital Francisco José de Caldas (UDFJC), \\ Bogotá, Colombia \\ ${ }^{2}$ Department of Communication and Information Science, MSc Program, Universidad Distrital Francisco José de Caldas (UDFJC), \\ Bogotá, Colombi
}

\section{Article Info \\ Article history: \\ Received Jun 30, 2021 \\ Revised Nov 17, 2021 \\ Accepted Dec 1, 2021}

\section{Keywords:}

Fusion

Gram schmidt

High frequency modulation

PCA

Satellite images

Simple mean value

Wavelet 2D-M

\begin{abstract}
Image-fusion provide users with detailed information about the urban and rural environment, which is useful for applications such as urban planning and management when higher spatial resolution images are not available. There are different image fusion methods. This paper implements, evaluates, and compares six satellite image-fusion methods, namely wavelet 2D-M transform, gram schmidt, high-frequency modulation, high pass filter (HPF) transform, simple mean value, and PCA. An Ikonos image (PanchromaticPAN and multispectral-MULTI) showing the northwest of Bogotá (Colombia) is used to generate six fused images: MULTIwavelet 2D-M, MULTI $_{\mathrm{G}-S}$, MULTI $I_{\mathrm{MHF}}$, MULTI $\mathrm{HPF}_{\mathrm{HP}}$, MULTISMV, and MULTIPCA. In order to assess the efficiency of the six image-fusion methods, the resulting images were evaluated in terms of both spatial quality and spectral quality. To this end, four metrics were applied, namely the correlation index, erreur relative globale adimensionnelle de synthese (ERGAS), relative average spectral error (RASE) and the Q index. The best results were obtained for the MULTIsmv image, which exhibited spectral correlation higher than 0.85 , a $\mathrm{Q}$ index of 0.84 , and the highest scores in spectral assessment according to ERGAS and RASE, $4.36 \%$ and $17.39 \%$ respectively.
\end{abstract}

This is an open access article under the CC BY-SA license.

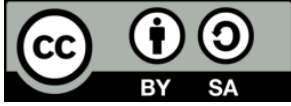

\section{Corresponding Author:}

Javier Medina

Department of Cadastral Engineering and Geodesy, School of Engineering

Universidad Distrital Francisco José de Caldas

Carrera 7, No. 40b-53, Bogotá DC, Colombia

Email: rmedina@udistrital.edu.co

\section{INTRODUCTION}

Image fusion is a solution that allows satisfying the frequent need for obtaining a single satellite image that carries both spectral (multi-spectral) and spatial (panchromatic) high-resolution data. The input to the fusion process is commonly taken from satellite images originated with the same type of sensor, or else, from several remote sensors so that decision makers are provided with the most effective basis available [1]-[12]. Image fusion delivers detailed information about rural and urban environments [13], which is useful for several applications such as territorial planning [14], agriculture [15], environmental monitoring [16], [17], medecine [18]-[21] among others.

Conventional image fusion processes are based on several methods such as RGB-to-IHS transformation, Brovey techniques, and multiplication, among others. However, these methods are not completely successful since spatial information is enhanced at the cost of degrading spectral information. Given this limitation, other processes have been explored. Processes involving the two-dimensional wavelet 
transform have been employed due to a reduced degradation of the spectral information contained in the original multi-spectral images, also leading to improvement in the spatial resolution [22]-[31]. According to wavelet-based studies, showing promising results for image fusion, [5], [26], [27], [32], [33] the wavelet transform contributes to obtaining better fused images due to the way in which coefficients are computed as part of the transformation process [34], [35]. This process yields the wavelet planes, which keep more spatial and spectral information from the original images than other techniques. Wavelet-based techniques also produce an intensity component that maintains the spatial richness of images along with tone and saturation components that that maintain spectral richness when transforming the image composition from RGB to IHS [36].

The present work focuses on six satellite-image fusion methods, namely the wavelet 2D-M transform using the RGB-to-HSV color model [37], the gram schmidt (G-S) method, high-frequency modulation (HFM), high pass filter transform (HPF), simple mean value (SMV), and principal component analysis (PCA). A step-by-step implementation process is proposed for applying each of the six methods. Moreover, a reference framework is established to assess the spectral and spatial quality of the resulting fused images (MULTI Wavelet 2D-M, MULTI $_{\mathrm{G}-S}$, MULTI $\mathrm{MHF}_{\mathrm{H}}, \mathrm{MULTI}_{\mathrm{HPF}}, \mathrm{MULTI}_{\mathrm{SMV}}$, y MULTI $\mathrm{MCA}_{\mathrm{A}}$ ) in terms of specific numerial indexes, namely the correlation coefficient, the relative average spectral error (RASE) index, the erreur relative globale adimensionnelle de synthese (ERGAS) index, and the Q universal quality index.

\section{METHODOLOGY}

This section is threefold: a description of the satellite images employed in the study is provided; followed by a description of the six image-fusion methods, which includes a proposal for implementing the methods; finally, the metrics applied to assess and compare the (spatial and spectral) quality of the resulting images are presented.

\subsection{Satellite images and the region covered}

The region covered by the satellite images corresponds to a western area of Bogotá (Colombia), specifically the area around the airport of the city, namely "El Dorado" airport. This region is covered by Ikonos of sub-images multi-spectral (MULTI) origin as shown in Figure 1(a) and panchromatic (PAN) as shown in Figure 1(b). The PAN sub-image has a spatial resolution of one (1) meter; this image was captured on December $13^{\text {th }}, 2007$ according to the UTM/WGS 84 reference system. The MULTI sub-image includes four-channel information; however, only three channels were involved in the present study (R-red, G-green and B-blue). The image has a spatial resolution of four (4) meters and was captured on the same date as the PAN sub-image, also sharing the same reference system. The two images were cropped to have a width of 2048 pixels and a height of 2048 pixels, satisfying dyadic properties [23].

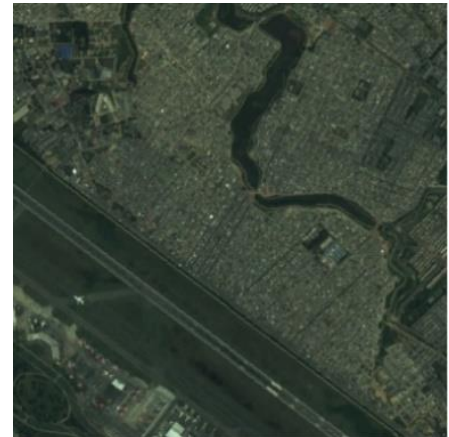

(a)

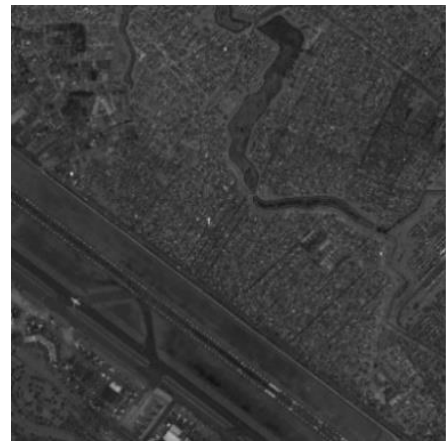

(b)

Figure 1. Original images are (a) MULTI Sub-image and (b) PAN sub-image; 2048x2048 pixels

\subsection{D wavelet transform}

The wavelet discrete transform is a useful tool in the field of signal processing. This transform is mainly applied to divide data sets into smaller components associated to different spatial frequencies, which are represented by a common scale. Algorithms known as Mallat and 'à trous' are typical in applying the discrete wavelet transform to image fusion. Each of these algorithms has its own mathematical properties and leads to a different type of image decomposition; therefore, different types of fused images can be expected. Although the 'á trous' algorithm appears to be less adequate than the Mallat algorithm when extracting spatial details in the context of multiresolution analysis (from a theoretical perspective), this algorithm yields 
images with significantly higher global quality [38]. For the six methods applied in this study, an RGB (true) color composition must be rendered from a combination of the MULTI and the PAN sub-images, using the same pixel size of the latter (1 meter).

Procedure for implementing 2D-M wavelet transform, (A modification to the wavelet haar transform) [33], [34]:

- Step 1. Transform the RGB image into hue, saturation, value (HSV) and adjust the histograms of the PAN image and the $\mathrm{V}$ component, yielding the new Vap component.

- Step 2. Apply the 2D-M wavelet transform to the V component (second decomposition level), yielding the corresponding approximation and detail coefficients; the A1v approximation coeficientes contain the spectral information of the image; meanwhile, detail coefficients $\mathrm{cV} 1 \mathrm{v}, \mathrm{cH} 1 \mathrm{v}$ and $\mathrm{cD} 1 \mathrm{v}$ store the spatial information of the image. Decompose the $\mathrm{A} 1 \mathrm{v}$ a second time, yielding the A2v approximation coefficients that contain the spectral information of the image; meanwhile, $\mathrm{cV} 2 \mathrm{v}, \mathrm{cH} 2 \mathrm{v}$ and $\mathrm{cD} 2 \mathrm{v}$ along with $\mathrm{cV} 1 \mathrm{v}, \mathrm{cH} 1 \mathrm{v}$ and $\mathrm{cD} 1 \mathrm{v}$ correspond to the detail coefficients that hold the spatial information of the transformed image.

- Step 3. Apply the 2D-M wavelet transform to the panchromatic image (second decomposition level), yielding the corresponding approximation and detail coefficients; the A1p approximation coefficients contain the spectral information of the image; meanwhile, detail coefficients $\mathrm{cV} 1 \mathrm{p}, \mathrm{cH} 1 \mathrm{p}$ and $\mathrm{cD} 1 \mathrm{p}$ store the spatial information. Decompose A1p a second time to obtain the A2p second-level approximation coefficients, which contain the spectral information; meanwhile, $\mathrm{cV} 2 \mathrm{p}, \mathrm{cH} 2 \mathrm{p}$ and $\mathrm{cD} 2 \mathrm{p}$ along with $\mathrm{cV} 1$, $\mathrm{cH} 1 \mathrm{p}$ and $\mathrm{cD} 1 \mathrm{p}$ correspond to the detail coefficients bearing the spatial information of the transformed image.

- Step 4. Generate a new value component (NI), using the A2v coefficients that store the information of the $\mathrm{V}$-component image together with the second-level detail coefficients of the panchromatic image (cV2p, $\mathrm{cH} 2 \mathrm{p}$ and $\mathrm{cD} 2 \mathrm{p}$ ) and the detail coefficients from the first-level decomposition (cV1p, $\mathrm{cH} 1 \mathrm{p}$ and $\mathrm{cD} 1 \mathrm{p})$.

- Step 5. Apply the inverse 2D-M wavelet transform to obtain the new intensity component (NV).

- Step 6. Given the new NV, and the original hue and saturation components, generate the new HS-NV.

- Step 7. Conduct the inverse image transformation from HS-NV to RGB. Thus, the new multispectral image is obtained, which maintains the spectral resolution, leading to a gain in spatial resolution.

\subsection{Gram-schmidt}

The Gram-Schmidt image-fusion method is based on a general vector orthogonalization algorithm. The algorithm consists in taking non-orthogonal vectors and apply rotation to make them orthogonal. When applied to image processing, each band (the panchromatic, the red, the green, the blue and the infrared) corresponds to an $\mathrm{n}$-dimensional vector $(\#$ dimensions $=$ \# pixels).

Implementation procedure:

- Step 1. Simulate the panchromatic band from the low-spatial-resolution spectral bands.

- Step 2. Apply the Gram-Schmidt transform to the simulated panchromatic band and also to the spectral bands, employing the simulated panchromatic band as the first band.

- Step 3. Intercambiar la alta resolución espacial de banda pancromática con la primera banda de GramSchmidt.

- Step 4. Apply the inverse Gram-Schmidt transform to construct the high-resolution spectral bands.

\subsection{High-frequency modulation}

This method is a variation of the so-called spatial-domain fusion methods [39], which focus on transferring the high frequencies of a high-resolution image onto a low-resolution image. The high-frequency representation of an image contains the information related to the details and such information can be obtained by means of filtering operators or convolution. Basically, these methods consist in adding the highfrequency components of the panchromatic image to each of the bands of a multi-spectral image:

$$
R_{i}=(M S)_{i}+P A(\text { Pan })
$$

where $R_{i}$ represents a generic band in the fusion and PA(Pan) is the result of applying a high-pass filter to the panchromatic image. The operation in (1) is similar to that of applying an enhancement filter to the high frequency components of an image (high-boost), although with a different image providing the high-pass information, namely the high-resolution panchromatic image in this case. Thus, the efficiency of this filter is based on the existence of radiometric correlation, which arises from the high-frequency components of the two images.

Implementation procedure:

- Step 1. Apply the high-pass filter and the low-pass filter to the panchromatic image. 
- Step 2. In order to generate each of the fused images, add each band to the product obtained from dividing the band over the low-pass filtered image times the high-pass filtered image.

- Step 3. Concatenate the fused images to obtain the new RGB image.

\subsection{High-pass filter transform}

This method consists in adding the spatial information from the panchromatic band to the multispectral information that has lower spatial resolution [40]. To this end, a high-pass filter is applied along with a map-algebra operation [41].

Implementation procedure:

- Step 1. Obtain a constant $\mathrm{R}$ by dividing the multi-spectral image cell size into the panchromatic image cell size.

- Step 2. Obtain a constant $\mathrm{R}$ by dividing the multi-spectral image cell size into the panchromatic image cell size. Based on this constant, a high-pass filter is generated and applied to the panchromatic image, yielding the standard deviations of both the multispectral image and the panchromatic image. A coefficient $\mathrm{W}$ is obtained, resulting from dividing the sum of the standard deviations into the product of the panchromatic standard deviation times the central value (M) as a function of the values of R [40].

- Step 3. Obtain the fused images, each image results from the addition of its corresponding band and the filtered image times $\mathrm{W}$.

- Step 4. Concatenate the fused images to obtain the new RGB image.

\subsection{Simple mean value}

This method applies a simple average equation to each of the combinations of output bands as:

- High-resolution Red Band $=0.5 *$ (original Red Band + Panchromatic Band)

- High-resolution Blue Band $=0.5 *$ (original Blue Band + Panchromatic Band)

- High-resolution Green Band = $0.5 *$ (original Green Band + Panchromatic Band)

Implementation procedure:

- Step 1. Generate fused images from the average of the Simple Mean Value, which consists of the sum of a single band and the panchromatic band (a result devided by 2), thus obtaining the fused images.

- Step 2. Concatenate the fused bands so as to obtain the new fused RGB image.

\subsection{PCA}

The principal component analysis, also coined PCA transformation, Karhunen-Loève transform, or Hotelling transform [42], is intended to create new images (Principal Components-PC) from the original images; such Principal Components (PCs) are non-correlated and lead to a reorganization of the original information. By having the PCs, redundant information is avoided. Thus, the first PC is defined as the direction of maximum data variance. The essence of this analysis is the transformation of a set of correlated variables into a new set of non-correlated variables [43].

Implementation procedure:

- Step 1. Obtain as many principal components as the number of bands in the multi-spectral image. Thus, PC1 holds spatial information, and the remaining PCs contain the spectral information.

- Step 2. Equate the histogram from the panchromatic image to that of the first principal component (PC1), that is, to the component that holds information related to the set of bands.

- Step 3. Substitute the first principal component (PC1) with the modified panchromatic image (once the histogram has been adjusted).

- Step 4. Apply the inverse transform to the resulting components so as to obtain the fused image, namely the new, fused RGB image.

\subsection{Assessment metrics}

For the evaluation and analysis of the resulting fused images obtained by means of MULTI $\mathrm{Wavelet}_{2 \mathrm{D}-}$ M, MULTI ${ }_{\mathrm{G}-\mathrm{S}}, \mathrm{MULTI}_{\mathrm{MHF}}, \mathrm{MULTI}_{\mathrm{HPF}}$, MULTI $\mathrm{SMV}$, and MULTIPCA, the following indexes were computed: the correlation coefficient (corr), the relative average spectral error (RASE) index, the ERGAS index (erreur relative globale adimensionallede synthèse) and the universal quality index Q. This set of indexes is described below.

i) Correlation coefficient (corr). The correlation between pairs of bands $\operatorname{corr}(A / B)$ from a fused image and the bands of an original image can be computed as (2):

$$
\operatorname{corr}(A / B)=\frac{\sum_{j=1}^{n p i x}\left(A_{j}-\bar{A}\right)\left(B_{J}-\bar{B}\right)}{\sqrt{\sum_{j=1}^{n p i x}\left(A_{j}-\bar{A}\right)^{2} \sum_{j=1}^{n p i x}\left(B_{j}-\bar{B}\right)^{2}}}
$$


Where $\bar{A}$ and $\bar{B}$ are the mean values of the two images. $\operatorname{corr}(A / B)$ can take values between -1 and +1 and it has no units as shown in Table 1, that is, the coefficient does not depend on the units of the original variables. The ideal value of the correlation coefficient, for both spectral and spatial assessment, is 1 [44].

ii) ERGAS index. The evaluation of the quality of fused images has been conducted by observing the value of the ERGAS indexes obtained for the spectral and spatial features as shown in Table 2. The spectral ERGAS index is defined in (3) [45]:

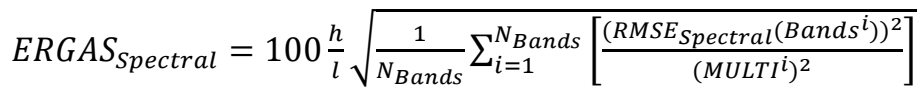

Where $h$ and $l$ represent the spatial resolution of the PAN and MULTI images; NBands corresponds to the number of bands of the fused image; $M U L T I^{i}$ is the radiance value associated to the $i$-ésima band of the MULTI image [46], and RMSE is defined in (4):

$$
R M S E_{\text {Espectral }}\left(\text { Banda }_{i}\right)=\frac{1}{N P} \sqrt{\sum_{i=1}^{N P}\left(M U L T I_{i}-F U S_{i}\right)^{2}}
$$

In (4), NP denotes the number of pixels of the $F_{U S}{ }^{i}(x, y)$ image. Moreover, in [47], an index coined ERGAS $S_{\text {Espacial }}$ is proposed. This index follows the same principles of the ERGAS index. $E R G A S_{\text {Espacial }}$ is aimed at evaluating the spatial quality of fused images and is defined in (5):

$$
E R G A S_{\text {Spatial }}=100 \frac{h}{l} \sqrt{\frac{1}{N_{\text {Bands }}} \sum_{i=1}^{N_{\text {Bands }}\left[\frac{\left(R M S E_{\text {Spacial }}\left(\text { Bands }^{i}\right)\right)^{2}}{\left(P A N^{i}\right)^{2}}\right]}}
$$

Where $R M S E_{\text {Espacial }}$ is a term defined in (6):

$$
R M S E_{\text {Espacial }}\left(\text { Banda }_{i}\right)=\frac{1}{N P} \sqrt{\sum_{i=1}^{N P}\left(P A N_{i}-F U S_{i}\right)^{2}}
$$

After evaluation of image quality usign these indexes (i.e. spatial and spectral ERGAS), values close to zero indicate excellent quality (see Table 2).

RASE index. The RASE index is expressed as a percentage (7), as indicated by the results in Table 2:

$$
R A S E=100 \frac{h}{l} \sqrt{\frac{1}{N} \sum_{i=1}^{n}\left[\frac{\left(R M S E\left(B_{i}\right)\right)^{2}}{M_{i}^{2}}\right]}
$$

Where $h$ is the value of resolution associeted to the high-spatial-resolution (PAN) image, and $l$ is the value of resolution associated to the low-spatial-resolution (MULTI) image [46]. The best results are obtained when the percentage indicated by RASE goes to zero.

Universal quality $\mathrm{Q}$ index. This quality index is intended to identify image distortion as a combination of three factors, namely correlation loss, luminance distortion and contrast distortion [48]. This index is copmputed according to (8) (see results in Table 2).

$$
Q=\frac{\sigma_{x y}}{\sigma_{x} \sigma_{y}} \cdot \frac{2 \bar{x} \bar{y}}{(\bar{x})^{2}+(\bar{y})^{2}} \cdot \frac{2 \sigma_{x} \sigma_{y}}{\sigma_{x}^{2}+\sigma_{x}^{2}}
$$

A higher quality of the fused image will be obtained as the value of Q approaches 1 .

\section{RESULTS AND DISCUSSION}

For qualitative assessment purposes, a visual inspection of the images is provided in Figure 2. The figure shows segments of the MULTI as shown in Figure 2(a) and PAN MULTI Figure 2(b) images as well as segments of the fused images obtained after applying the image fusion methods Figure 2(c)-(h). In addition to the visual assessment provided in Figure 2, quantitative results are presented in Tables 1 and 2. Table 2 shows the results that describe the spatial and spectral correlation of fused images after applying the six methods. 


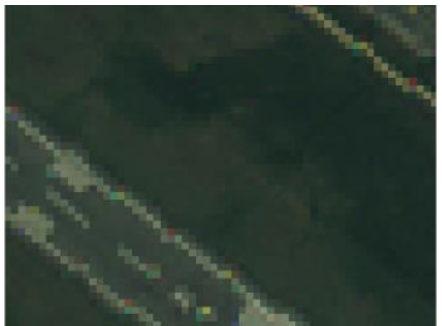

(a)

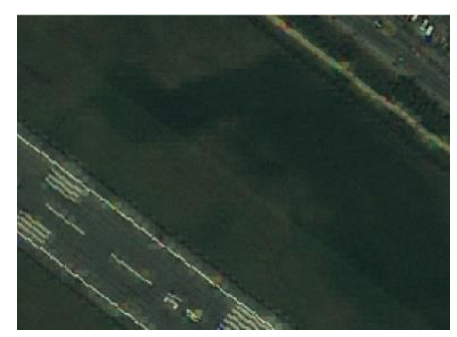

(c)

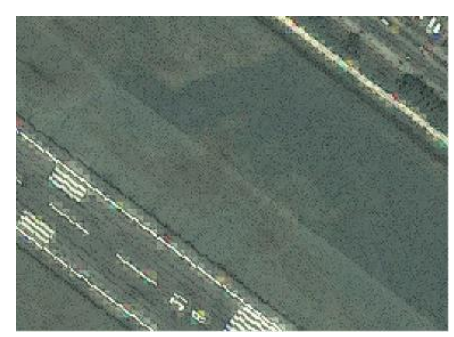

(e)

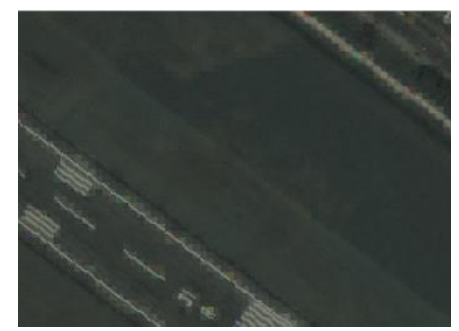

(g)

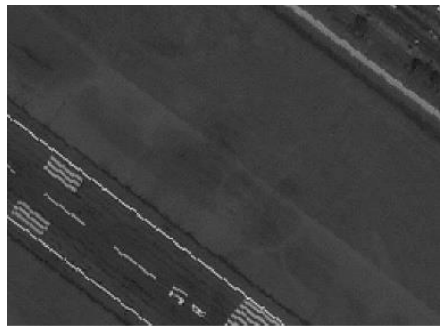

(b)

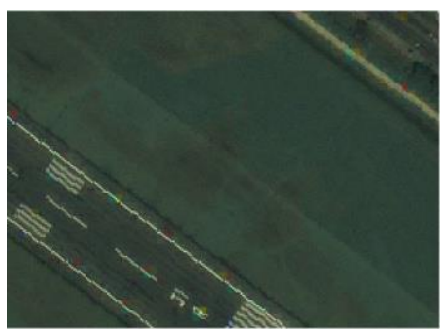

(d)

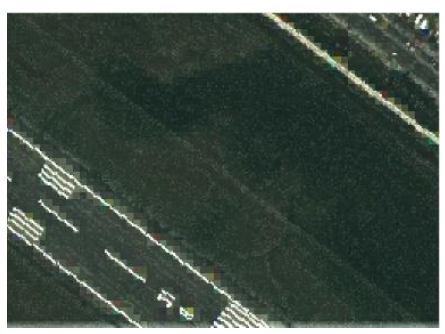

(f)

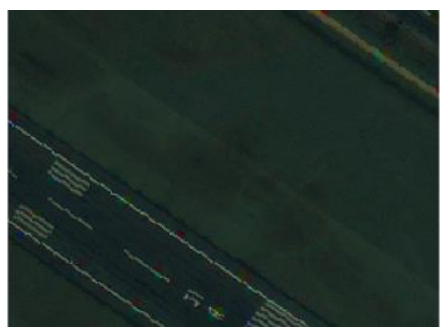

(h)

Figure 2. Detail of sub-images; (a) MULTI, (b) PAN, (c) Wavelet fused, d) Gram-schmitd fused, (e) High-frequency modulation, (f) HPF fused, (g) Simple mean value fused, and (h) PCA fused

Table 1. Quantitative evaluation of Ikonos image quality, spatial and spectral correlation

\begin{tabular}{lcccccc}
\hline \multicolumn{1}{c}{ Fused Image } & \multicolumn{3}{c}{ corr spatial } & \multicolumn{3}{c}{ corr spectral } \\
& $\mathrm{R}$ & $\mathrm{G}$ & $\mathrm{B}$ & $\mathrm{R}$ & $\mathrm{G}$ & $\mathrm{B}$ \\
\hline MULTI $_{\text {Wavelet } 2 \mathrm{D}-\mathrm{M}}$ & 0.77 & 0.81 & 0.77 & $\mathbf{0 . 8 5}$ & $\mathbf{0 . 7 9}$ & $\mathbf{0 . 7 6}$ \\
MULTI $_{\text {G-S }}$ & $\mathbf{0 . 9 2}$ & $\mathbf{0 . 9 7}$ & $\mathbf{0 . 9 5}$ & 0.78 & 0.69 & 0.62 \\
MULTI $_{\text {MAF }}$ & 0.66 & 0.68 & 0.65 & $\mathbf{0 . 9 4}$ & $\mathbf{0 . 9 2}$ & $\mathbf{0 . 9 0}$ \\
MULTI $_{\text {HPF }}$ & 0.53 & 0.54 & 0.49 & 0.70 & 0.69 & 0.60 \\
MULTI $_{\text {SMV }}$ & 0.84 & 0.85 & 0.88 & 0.88 & 0.87 & 0.80 \\
MULTI $_{\text {PCA }}$ & $\mathbf{0 . 9 4}$ & $\mathbf{0 . 9 7}$ & $\mathbf{0 . 8 9}$ & 0.55 & 0.54 & 0.57 \\
\hline
\end{tabular}

In terms of the spatial correlation of images, the results show that the highest values of the coefficients are obtained for the MULTI $_{\mathrm{G}-\mathrm{S}}$, MULTI ${ }_{\mathrm{PCA}}$ images in each of the three bands. Regarding the spectal correlation of images, the highest values can be observed for the MULTI Wavelet 2D-M, MULTI MAF $_{\text {fused }}$ images. Table 2 shows the resulting values of computing the RASE index, the ERGAS index and the universal quality $\mathrm{Q}$ index, regarding both spatial and spectral features. 
Table 2. Quality evaluation for the Ikonos sub-image, values of ERGAS, RASE, and Q indexes, spatial and spectral evaluation

\begin{tabular}{ccccccc}
\hline Fused image & ERGAS spatial & ERGAS spectral & RASE spatial & RASE spectral & Q spatial & Q spectral \\
\hline MULTI $_{\text {Wavelet } 2 \mathrm{D}-\mathrm{M}}$ & 6.22 & 5.38 & $24.89 \%$ & $21.60 \%$ & 0.77 & 0.79 \\
MULTI $_{\mathrm{G}-\mathrm{S}}$ & $\mathbf{3 . 6 3}$ & $\mathbf{5 . 9 7}$ & $\mathbf{1 4 . 5 2} \%$ & $\mathbf{2 4 . 0 0} \%$ & $\mathbf{0 . 9 3}$ & $\mathbf{0 . 7 0}$ \\
MULTI $_{\mathrm{MAF}}$ & 8.92 & 5.22 & $35.68 \%$ & $20.96 \%$ & 0.64 & 0.89 \\
MULTI $_{\mathrm{HPF}}$ & 15.32 & 13.93 & $61.31 \%$ & $55.01 \%$ & 0.41 & 0.52 \\
MULTI $_{\mathrm{SMV}}$ & $\mathbf{4 . 4 2}$ & $\mathbf{4 . 3 6}$ & $\mathbf{1 7 . 7 1} \%$ & $\mathbf{1 7 . 3 9} \%$ & $\mathbf{0 . 8 4}$ & $\mathbf{0 . 8 4}$ \\
MULTI $_{\mathrm{PCA}}$ & 11.255 .38 & 12.48 & $45.01 \%$ & $49.99 \%$ & 0.70 & 0.43 \\
\hline
\end{tabular}

The results obtained by computing the spatial ERGAS index highlight the quality of the MULTI $\mathrm{G}_{-\mathrm{S}}$ fused image (spatial ERGAS equal to 3.63). Meanwhile, the spectral ERGAS index indicates that the

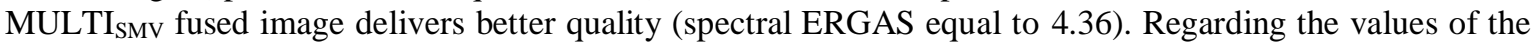
spatial and spectral RASE indexes, the best results follow the same trend (as observed for ERGAS), that is, the most promising values of spatial RASE are associated to the MULTI $\mathrm{G}_{\mathrm{G}-\mathrm{S}}$ image $(14.52 \%$, spatial and $24 \%$ spectral) and the best spectral RASE score was obtained for the MULTISMV image (17.71\%). Finally, the values of the $\mathrm{Q}$ index (spatial and spectral) show excellent results for both the MULTI $\mathrm{G}_{\mathrm{M} M}$ image and the MULTI $_{\text {SMv }}$ image; however, the MULTI $I_{\text {SMv }}$ fused image outperforms the others since the two indexes (spectral and spactial) maintain equivalent values.

Previous work [1], [5], [26], [27], [32], [33], [35], [37], [38], [41], [43], [47] has shown that image fusion methods based on the wavelet transform (employing the Á trous and Mallat algorithms) are better suited for the fusion of images than traditional methods. Indexes:

Moreover, Wald [45] proposed a set of requirements that should be met by quality tests and Quality

1. Indexes should be unit-independent, including calibration instuments and gain values.

2. Indexes should not depend on number of spectral bands.

3. Indexes should not depend on the relation among the spatial resolution of the source images.

Therefore, the results provided for the qualitative analysis in Figure 2 and the quantitative analysis (Tables 1 and 2) show that the best fused image (the image resulting in the smallest degradation of spectral richness with significant spatial gain) was obtained when applying the Simple Mean Value algorithm (MULTISMV).

\section{CONCLUSION}

The implementation of various mathematical proposals, namely 2D-M wavelet transform, Gram Schmidt transform, High-frequency modulation, HPF transform, Simple Mean Value and Principal Component Analysis, allowed generating fused images from the satellite Ikonos images that are useful for a variety of applications. Implementation of various methods in a single study favors the interest of researchers, since the best method has not been found, instead, the best fused image must be found. The best fused image showing the highest spatial gain, though with some degradation of spectral richness, is the MULTI G-S $_{\text {image. The Simple Mean Value method (MULTI }}$ smv) allowed fused images with higher spatial and spectral quality; however, it can be concluded that the images with higher spatial quality tend to degrade spectral richness, conversely, the images with higher spectral quality fail to offer excellent spatial features. The methods proposed herein lead to fused images that provide users with detailed information about urban and rural surroundings, which is useful for applications such as urban planning and management when there is no availability of higher spatial resolution images (e.g. drone-based images). The usefulness of the present study also extends to project development in fields such as agriculture, hydrology, environmental sciences and natural dissarter scenarios (e.g. floods, forrest fires), among others.

\section{REFERENCES}

[1] L. Alparone, L. Wald, J. Chanussoat, C. Thomas, P. Gamba, and L. Bruce, "Comparison of Pansharpening Algorithms: Outcome of the 2006 GRS-S Data Fusion Contest," IEEE Transactions on Geoscience and Remote Sensing, vol. 45, no. 10, pp. 3012-3021, 2007, doi: 10.1109/TGRS.2007.904923.

[2] A. A. Goshtasby and S. Nikolov, "Image Fusion: Advances in the State of the Art," Information Fusion, vol. 8, pp. 114-118, 2007.

[3] D. Wu, A. Yang, L. Zhu, and C. Zhang, "Survey of Multi-sensor Image Fusion," S. Ma et al. (Eds.): LSMS/ICSEE 2014, Part I, CCIS 461, pp. 358-367, 2014, doi: 10.1007/978-3-662-45283-7_37.

[4] H. Ghassemian, "A Review of Remote Sensing Image Fusion Methods,” Information Fusion, 32, Part A, pp. 75-89, 2016, doi: 10.1016/j.inffus.2016.03.003.

[5] J. Jinju, N. Santhi, K. Ramar, and B. Sathya Bama, "Spatial frequency discrete wavelet transform image fusion technique for remote sensing applications," Engineering Science and Technology, an International Journal, vol. 22, no. 3, pp. 715-726, 2019, doi: 10.1016/j.jestch.2019.01.004. 
[6] Z. W. Pan and H. L. Shen, "Multispectral Image super-resolution via RGB image fusion and radiometric calibration," IEEE Transactions on Image Processing, vol. 28, no. 4, pp. 1783-1797, 2019, doi: 10.1109/TIP.2018.2881911.

[7] S. C. Kulkarni and P. P. Rege, "Pixel level fusion techniques for SAR and optical images: A review," Information Fusion, vol. 59, pp. 13-29, 2020, doi: 10.1016/j.inffus.2020.01.003.

[8] R. V. Pandit and R. J. Bhiwani, "Morphology-based spatial filtering for efficiency enhancement of remote sensing image fusion," Computers \& Electrical Engineering, vol. 89, p. 106945, 2021, doi: 10.1016/j.compeleceng.2020.106945.

[9] M. Diwakar et al., "A comparative review: Medical image fusion using SWT and DWT," Materials Today: Proceedings, vol. 37, no. Part 2, pp. 3411-3416, 2021, doi: 10.1016/j.matpr.2020.09.278.

[10] S. Singh and K. C. Tiwari, "Exploring the optimal combination of image fusion and classification techniques," Remote Sensing Applications: Society and Environment, vol. 24, p. 100642, 2021, doi: 10.1016/j.rsase.2021.100642.

[11] X. Zhang, "Benchmarking and comparing multi-exposure image fusion algorithms," Information Fusion, vol. 74, pp. 111-131, 2021, doi: 10.1016/j.inffus.2021.02.005.

[12] C. S. Yilmaz, V. Yilmaz, and O. Gungor, "A theoretical and practical survey of image fusion methods for multispectral pansharpening," Information Fusion, vol. 79, pp. 1-43, 2022, doi: 10.1016/j.inffus.2021.10.001.

[13] C. Nietupski Ty, R. E. Kennedy, H. Temesgen, and B. K. Kerns, "Spatiotemporal image fusion in Google Earth Engine for annual estimates of land surface phenology in a heterogenous landscape," International Journal of Applied Earth Observation and Geoinformation, vol. 99, p. 102323, 2021, doi: 10.1016/j.jag.2021.102323.

[14] S. Wu and H. Chen, "Smart city oriented remote sensing image fusion methods based on convolution sampling and spatial transformation," Computer Communications, vol. 157, pp. 444-450, 2020, doi: 10.1016/j.comcom.2020.04.010.

[15] D. Li, Z. Song, C. Quan, X. Xu, and C. Liu, "Recent advances in image fusion technology in agriculture," Computers and Electronics in Agriculture, vol. 191, p. 106491, 2021, doi: 10.1016/j.compag.2021.106491.

[16] J. Kong et al., "Evaluation of four image fusion NDVI products against in-situ spectral-measurements over a heterogeneous rice paddy landscape," Agricultural and Forest Meteorology, vol. 297, p. 108255, 2021, doi: 10.1016/j.agrformet.2020.108255.

[17] Z. Cao, S. Chen, F. Gao, and X. Li, "Improving phenological monitoring of winter wheat by considering sensor spectral response in spatiotemporal image fusion," Physics and Chemistry of the Earth, Parts $A / B / C$, vol. 116, p. 102859, 2020, doi: 10.1016/j.pce.2020.102859.

[18] Y. Li, J. Zhao, Z. Lv, and J. Li, "Medical image fusion method by deep learning," International Journal of Cognitive Computing in Engineering, vol. 2, pp. 21-29, 2021, doi: 10.1016/j.ijcce.2020.12.004.

[19] H. Xu and J. Ma, "EMFusion: An unsupervised enhanced medical image fusion network," Information Fusion, vol. 76, pp. 177-186, 2021, doi: 10.1016/j.inffus.2021.06.001.

[20] X. Li, F. Zhou, and H. Tan, "Joint image fusion and denoising via three-layer decomposition and sparse representation," Knowledge-Based Systems, vol. 224, p. 107087, 2021, doi: 10.1016/j.knosys.2021.107087.

[21] G. Li, Y. Lin, and X. Qu, "An infrared and visible image fusion method based on multi-scale transformation and norm optimization," Information Fusion, vol. 71, pp. 109-129, 2021, doi: 10.1016/j.inffus.2021.02.008.

[22] M. Misiti, Y. Misiti, G. Oppenheim, and J.-M. Poggi, Wavelets toolbox For use with Matlab, The Maht works Inc, Natick, MA 01760-2098, Version 4.4.1., 2009.

[23] Y. Nievergelt, Wavelets made easy, Ed Birkhäuser, New York, 2013, doi: 10.1007/978-1-4614-6006-0.

[24] C. Burrus, Wavelets and Wavelet Transforms, Sep. 24, 2015, http://cnx.org/content/col11454/1.6/

[25] G. Siddalingesh, A. Mallikarjun, H. Sanjeevkumar, and S. Kotresh, "Feature-Level Image Fusion Using DWT, SWT, and DTCWT," Emerging Research in Electronics, Computer Science, and Technology, Lecture Notes in Electrical Engineering, vol. 248, pp. 183-194, 2014, doi: 10.1007/978-81-322-1157-0_20.

[26] N. Jha, A. K. Saxena, A. Shrivastava, and M. Manoria, "A review on various image fusion algorithms," 2017 International Conference on Recent Innovations in Signal Processing and Embedded Systems (RISE), Oct. 2017, pp. 27-29, doi: $10.1109 /$ rise.2017.8378146.

[27] C. Periyasamy, "Satellite Image Enhancement Using Dual Tree Complex Wavelet Transform," Bulletin of Electrical Engineering and Informatics, vol. 6, no. 49, pp. 334-336, 2017, doi: 10.11591/eei.v6i4.861.

[28] A. Sharma and T. Gulati, "Change Detection from Remotely Sensed Images Based on Stationary Wavelet Transform," International Journal of Electrical and Computer Engineering, vol. 7, no. 6, pp. 3395-3401, 2017, doi: 10.11591/ijece.v7i6.pp3395-3401.

[29] V. Radhika, K. Veeraswamy, and S. S. Kumar, "Digital Image Fusion Using HVS in Block Based Transforms," J Sign Process Syst, vol. 90, pp. 947-957, 2018, doi: 10.1007/s11265-017-1252-8.

[30] Y. Liu, L. Wang, J. Cheng, C. Li, and X. Chen, "Multi-focus image fusion: A Survey of the state of the art," Information Fusion, vol. 64, pp. 71-91, 2020, doi: 10.1016/j.inffus.2020.06.013.

[31] M. Jindal, E. Bajal, A. Chakraborty, P. Singh, M. Diwakar, and M. Kumar, "A novel multi-focus image fusion paradigm: A hybrid approach," Materials Today: Proceedings, vol. 37, Part 2, pp. 2952-2958, 2021, doi: 10.1016/j.matpr.2020.08.704.

[32] J. Nuñez, X. Otazu, O. Fors, A. Prades, V. Pala, and R. Arbiol, "Multiresolution-Based Image fusion whit Additive Wavelet Descomposition," IEEE Transactions on Geoscience and Remote Sensing, vol. 37, no. 3, pp. 1204-1211, 1999, doi: 10.1109/36.763274.

[33] J. Medina, C. Pinilla, and L. Joyanes, "Two-Dimensional Fast Haar Wavelet Transform for Satellite-Image Fusion,” Journal of Applied Remote Sensing, vol. 7, no. 1, p. 073698, 2013, doi: 10.1117/1.JRS.7.073698.

[34] E. Upegui and J. Medina, Análisis de Imágenes usando las trasformadas Fourier y Wavelet, Bogotá: Editorial Unioversidad Distrital Francisco José de Caldas, 2019, ISBN 978-787-064-0.

[35] C. Gonzalo and M. Lillo-Saavedra, "A directed search algorithm for setting the spectral-spatial quality trade-off of fused images by the wavelet à trous method," Canadian Journal of Remote Sensing, vol. 34, no. 4, pp. 367-375, 2008, doi: 10.5589/m08-041.

[36] R. C. González and R. E. Woods, Digital Image Processing, 4th. Edition, Pearson, 2018, ISBN: 9780133356779.

[37] J. Medina, I. Carrillo, and E. Upegui, "Implementación y evaluación de la transformada Wavelet 2D À trous: usando Intensidad, Luminancia y Value en Matlab para Fusión de Imágenes Satelitales,” RISTI, no. E41, pp. 396-409, 2021.

[38] C. Gonzalo-Martín and M. Lillo-Saavedra, "Fusión de Imágenes QuickBird Mediante una Representación Conjunta Multirresolución-Multidireccional," IEEE Latin America Transactions, vol. 5, no. 1, pp. 32-37, 2007, doi: 10.1109/TLA.2007.4444530.

[39] R. A. Schowengerdt, Remote Sensing. Models and Methods for image Processing, 3rd edition, Elseiver, 2013.

[40] Hexagon Geospatial, ERDAS Imagine 2018, Online Documentation, 2018.

[41] C. Pohl and J. L. Van Genderen, "Review Article, Structuring contemporary remote sensing image fusion," International Journal of Image and Data Fusion, vol. 6, no. 1, pp. 3-21, 2015, doi: 10.1080/19479832.2014.998727. 
[42] K. Shettigara, “A Linear Transformation Technique For Spatial Enhancement Of Multispectral Images Using A Higher Resolution Data Set," 12th Canadian Symposium on Remote Sensing Geoscience and Remote Sensing Symposium, July, 1989, doi: 10.1109/IGARSS.1989.577945.

[43] R. J. Medina-Daza, N. E. Vera-Parra, and A. O. Restrepo-Rodríguez, "Sallfus, Library for Satellite Images Fusion on Homogeneous and Heterogeneous Computing architectures," IEEE Latin America Transactions, vol. 18, no. 12, pp. 2130-2137, 2020, doi: 10.1109/TLA.2020.9400441.

[44] S. R. Murray and J. F. Larry, Estadística, Cuarta edición, Mc Graw Hill, 2009.

[45] L. Wald, Data Fusion, Definition and Architectures: Fusion of Image of Different Spatial Resolution, Le Presses de 1'Ecole des Mines, Paris, 2002.

[46] L. Wald, "Quality of high resolution synthesized images: is there a simple criterion?," Proceedings of the third conference Fusion of Earth data: merging point measurements, raster maps and remotely sensed image, Sophia Antipolis, France, January 26-28, 2000.

[47] M. Lillo-Saavedra, C. Gonzalo, A. Arquero, and E. Martinez, "Fusion of multispectral and panchromatic satellite sensor imagery based on tailored filtering in the Fourier domain," International Journal of Remote Sensing, vol. 26, pp. 1263-1268, 2005, doi: 10.1080/01431160412331330239.

[48] Z. Wang and A. C. Bovink, “A Universal Image Quality Index,” IEEE Signal Processing Letters, vol. 9, no. 3, pp. 81-84, 2002, doi: $10.1109 / 97.995823$

\section{BIOGRAPHIES OF AUTHORS}

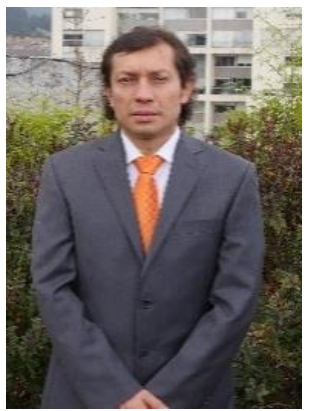

Javier Medina (ID SA SC P in Computer Science, emphasis on Geographic information systems, Pontifical University of Salamanca Madrid-Spain Campus. Accredited in research proficiency-Diploma Advanced Studies (DEA)-in Geographic information systems, Pontifical University of Salamanca Madrid-Spain campus. Master in Teleinformatics, Specialist in Software Engineering, Specialist in Geographic Information Systems and Bachelor of Mathematics from the Francisco José District University of Caldas. Full Professor Time complete assigned to the Faculty of Engineering from the same University, Teacher of the Curriculum Project Cadastral Engineering and Geodesy, Master in Information Sciences and Communications and the Doctorate of Engineering of the Faculty of Engineering of the Francisco José de Caldas. He can be contacted at email: rmedina@udistrital.edu.co.

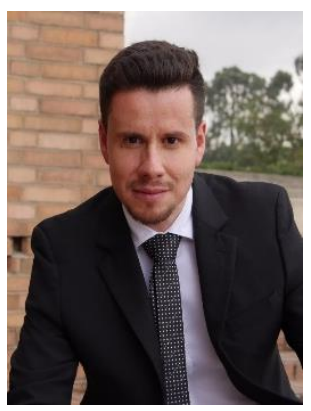

Nelson Vera (iD 81 SC P Professor and Coordinator of the master's program in information and communication sciences at the Universidad Distrital Francisco José de Caldas (Bogotá, Colombia), Doctor of Engineering from the same University, Electronic Engineer from the Universidad Surcolombiana (Neiva, Colombia), Researcher in parallel computing, high performance computing, science data and bioinformatics. He can be contacted at email: neverap@udistrital.edu.co.

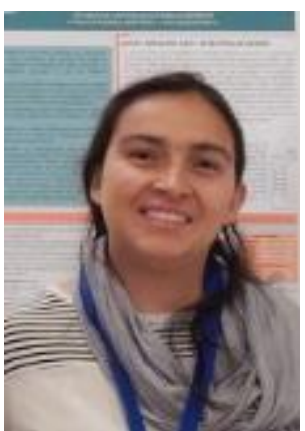

Erika Upegui (iD 8D SC P Associate Professor for the School of Engineering at Universidad Distrital Francisco José de Caldas. She is an active member of the Cadastral and Geodesy programs as well as the MSc program in Information and Communication sciences, and the $\mathrm{PhD}$ program in Engineering. She obtained her $\mathrm{PhD}$ title in Geography and Territorial Planning at Université Franche-Comté in France. She is MSc in Teledetection and Geomatics Aapplied to Environmental sciences at Université Paris 7 (France). She also holds the tite lof specialist in property appraisal and geographic information systems at Universidad Distrital Francisco José de Caldas (Colombia). She can be contacted at email: esupeguic@udistrital.edu.co. 